\title{
Neonatal Drug Withdrawal
}

National Cancer Institute

\section{Source}

National Cancer Institute. Neonatal Drug Withdrawal. NCI Thesaurus. Code C101321.

A constellation of neurobehavioral features observed following cessation or reduction of antenatal or postnatal drug exposure. 